\title{
Radiosinovectomía de muñeca guiada por ultrasonido: Experiencia de un caso clínico y revisión del tema
}

Drs. Oscar Contreras O, Pablo Riquelme M, Juan Quintana F, Jorge Llanos C, Álvaro Burdiles O.

Departamento de Radiología, Facultad de Medicina de la Pontificia Universidad Católica de Chile.

Ultrasound-guided wrist radiosynovectomy: Case report and topic review

Abstract: Radiosynovectomy is a local form of radiotherapy used as second-line treatment in the management of inflammatory and non-inflammatory arthropathies with unsatisfactory response to local or systemic corticosteroid therapy. Its efficacy is similar to that of surgical synovectomy, with the advantages of being a low-cost and minimally- invasive treatment that requires a shorter recovery time. Its efficacy is greater in the treatment of inflammatory arthritis characterized by synovitis, such as rheumatoid arthritis and juvenile chronic arthritis with mono/ oligoarticular involvement, especially in upper extremity joints. A number of isotopes can be used in colloidal suspensions. Rhenium-186-sulphide colloid is currently indicated for the treatment of medium-sized joints. The ultrasound-guided injection is suitable for small joints, such as wrist. For proper and safe use and administration of this technique the collaboration of a trained and experienced radiologist is critical to successful treatment.

Keywords: Juvenile chronic arthritis, Radiosynovectomy, Radiosynoviorthesis, Rhenium-186, Rheumatoid arthritis.

Resumen: La radiosinovectomía es una forma local de radioterapia utilizada como tratamiento de segunda línea en el manejo de artropatías inflamatorias y no inflamatorias con respuesta insatisfactoria a la terapia sistémica o local con corticoesteroides. Tiene una eficacia similar a la sinovectomía quirúrgica, con las ventajas de ser un tratamiento de menor costo, menos cruento y con menor tiempo de convalecencia. Su eficacia es mayor en artropatías inflamatorias caracterizadas por sinovitis, como la artritis reumatoide y artritis crónica juvenil, con compromiso mono u oligoarticular y en articulaciones de extremidades superiores. Existen múltiples isótopos que se pueden utilizar en suspensiones coloidales, siendo el sulfuro coloidal de renio-186 el utilizado para la articulación radiocarpiana. Una técnica de administración adecuada es fundamental para el éxito del tratamiento, siendo la inyección con guía ultrasonográfica adecuada para articulaciones de volumen pequeño, instancia en la que la colaboración del médico radiólogo entrenado es fundamental.

Palabras clave: Artritis crónica juvenil, Artritis reumatoide, Radiosinovectomía, Radiosinoviortesis, Renio-186.

Contreras $\mathrm{O}$, et al. Radiosinovectomía de muñeca guiada por ultrasonido: Experiencia de un caso clínico y revisión del tema. Rev Chil Radiol 2012; 18(3): 121-128.

Correspondencia: Dr. Álvaro burdiles / aburdiles@gmail.com

Trabajo recibido el 28 de julio de 2012, aceptado para publicación el 10 del septiembre de 2012.

Introducción

La radiosinovectomía (o radiosinoviortesis) es una técnica que se ha empleado desde la década de los ' $50^{(1)}$ para el tratamiento de sinovitis resistente a tratamiento sistémico en artropatías inflamatorias como la artritis reumatoide y en artropatía hemofílica. Consiste en una forma local de radioterapia basada en la inyección intraarticular de un radiofármaco emisor de partículas beta en forma de suspensión coloidal(2).

Tiene una tasa de éxito similar a la de la sinovectomía quirúrgica, con las ventajas de ser un procedimiento ambulatorio mínimamente invasivo, que requiere un menor tiempo de recuperación y que puede ser repetido ${ }^{(3)}$. Es especialmente útil en la artropatía hemofílica; la sinovectomía quirúrgica es un procedimiento complejo en estos pacientes, quienes requieren hospitalización prolongada y reemplazo de factores de la coagulación ${ }^{(4)}$.

La solución coloidal de renio-186 es la indicada actualmente para el tratamiento en articulaciones de mediano tamaño, como la cadera, tobillo, hombro y muñeca, dado que la profundidad de penetración de la emisión beta es la suficiente para la irradiación adecuada de la membrana sinovial, pero sin afectar el hueso subcondral ${ }^{(3)}$. 
La adecuada selección de pacientes y la estrecha colaboración entre los profesionales a cargo es fundamental para obtener los mejores resultados del tratamiento. Otro factor importante es la técnica adecuada de administración. Respecto a este punto se debe considerar la capacidad de distensión de la articulación a tratar, por lo que la guía ultrasonográfica presenta ventajas comparativas, ya que no es necesario inyectar medio de contraste para comprobar la posición intraarticular de la aguja (procedimiento que es necesario realizar cuando la inyección es bajo guía fluoroscópica).

El objetivo del presente trabajo es exponer la experiencia en nuestra institución en la realización de radiosinovectomía guiada bajo ultrasonido y presentar una revisión bibliográfica.

Indicaciones, selección y preparación de pacientes

La principal indicación para el uso de radiosinovectomía es como alternativa a la sinovectomía quirúrgica en sinovitis resistente al tratamiento farmacológico sistémico en pacientes con artropatías inflamatorias, más comúnmente la artritis reumatoide. También se benefician los pacientes con espondiloartropatías, enfermedad de Lyme, enfermedad de Behçet, otras artropatías inflamatorias caracterizadas por sinovitis (como la osteoartritis erosiva), sinovitis villonodular pigmentada, artropatía por depósito de pirofosfato de calcio, artropatía hemofílica y derrame articular persistente (incluyendo el derrame posterior a artroplastía) ${ }^{(2)}$. Al ser considerado un tratamiento de segunda línea, el paciente debe presentar dolor significativo a pesar del uso de analgésicos o limitación funcional.

Existen contraindicaciones absolutas (embarazo, lactancia, infección cutánea local y quiste poplíteo roto en caso de sinovitis de rodilla) y relativas (menores de 20 años, inestabilidad articular, destrucción extensa de cartílago y hueso subcondral) para el procedimiento ${ }^{(2)}$.

Es necesario considerar un intervalo de 6 semanas después de una cirugía articular y de 2 semanas después de una artrocentesis antes de realizar la radiosinovectomía, con el fin de limitar la posibilidad de filtración extraarticular del radiofármaco ${ }^{(3)}$. El procedimiento se puede repetir después de 6 meses, con una efectividad similar a la de la primera inyección ${ }^{(5)}$.

El paciente debe contar con estudios de imágenes previos recientes. Una radiografía suele ser suficiente, pero el estudio con resonancia magnética es recomendable para evaluar con mayor precisión el grado de sinovitis y de condropatía asociada ${ }^{(6)}$. El ultrasonido también es útil para evaluar la sinovitis y descartar la presencia de un quiste poplíteo roto en la rodilla. El cintigrama con difosfonato de $99 \mathrm{mT}$ se puede utilizar para documentar el número de articulaciones afectadas.

\section{Radiofármacos y técnica utilizada}

El radiofármaco elegido debe contar con algunas características. Se deben utilizar soluciones coloidales de tamaño suficiente para penetrar en el tejido sinovial (5 a 10 nanometros), la unión entre las partículas o coloide y el isótopo debe ser estable y por último, el fármaco debe distribuirse homogéneamente en la articulación sin causar una respuesta inflamatoria significativa ${ }^{(3)}$. Los isótopos utilizados habitualmente son el itrio-90 (Y90), el renio-186 (Re186) y el erbio-169 (Er169), emisores de partículas beta de vida media corta. El Y90 se administra como citrato o silicato coloidal y se utiliza únicamente en la rodilla. El Re186 se pude utilizar en cadera, hombro, tobillo, articulación subastragalina, codo y muñeca. Tiene la particularidad de emitir escasa radiación gamma, lo que permite realizar un estudio cintigráfico posterior para evaluar la distribución intrarticular adecuada del radiofármaco. El Er169 se utiliza en articulaciones pequeñas (metatarsofalángicas, metacarpofalángicas e interfalángicas) y se administra como citrato coloidal. En la tabla I se resumen las características de cada isótopo y se mencionan las dosis recomendadas para cada articulación, según las guías de la Asociación Europea de Medicina Nuclear ${ }^{(2)}$ (Tabla I. Radiofármacos utilizados en radiosinovectomía).

Tabla I. Radiofármacos utilizados en radiosinovectomía.

Radio- fármaco

Energía máxima /

Dosis recomendada por articulación promedio radiación beta

\begin{tabular}{|ll}
90 Y (Citrato o silicato coloidal) & $2,27 / 0,935 \mathrm{MeV}$ \\
186 Re (Sulfuro coloidal) & $\begin{array}{l}1,07 / 0,349 \mathrm{MeV} \\
\text { (también emite radiación } \\
\text { gamma de 137 KeV) }\end{array}$
\end{tabular}

$169 \mathrm{Er}$ (Citrato coloidal)

0,34 / 0,099 MeV

\author{
185-222 MBq / 5-6 mCi (rodilla)
}

74-185 MBq/ 2-5 mCi (cadera, hombro) $74-111 \mathrm{MBq} / 2 \mathrm{mCi}$ (codo)

37-74 MBq/ 1-2 mCi (muñeca; subastragalina) $74 \mathrm{MBq} / 2 \mathrm{mCi}$ (tobillo)

20-40 MBq/ 0,5-1 mCi (metacarpofalángicas) 30-40 MBq/ 0,8-1 mCi (metatarsofalángicas) 10-20 MBq/ 0,3-0,5 mCi (interfalángicas) 
La inyección debe realizarse por un profesional entrenado, con técnica estéril estricta y con medidas adecuadas de protección radiológica (Figura 1). Sólo la artrocentesis de rodilla se puede realizar sin guía flouroscópica o ecotomográfica. En el resto de las articulaciones el procedimiento debe ser guiado bajo imágenes para asegurar la inyección intraarticular(2). La inyección bajo guía ultrasonográfica por parte de un profesional entrenado tiene una serie de ventajas sobre la guía fluoroscópica, especialmente para articulaciones de pequeño o mediano tamaño como la muñeca ${ }^{(7)}$, entre las que se cuentan un menor volumen a inyectar, ya que no es necesario inyectar medio de contraste y por la posibilidad de evaluación diagnóstica concomitante, especialmente para evaluar el grado de sinovitis y la presencia de erosiones óseas. El menor volumen inyectado tiene la ventaja de disminuir el riesgo de fuga del radiofármaco hacia los tejidos periarticulares.

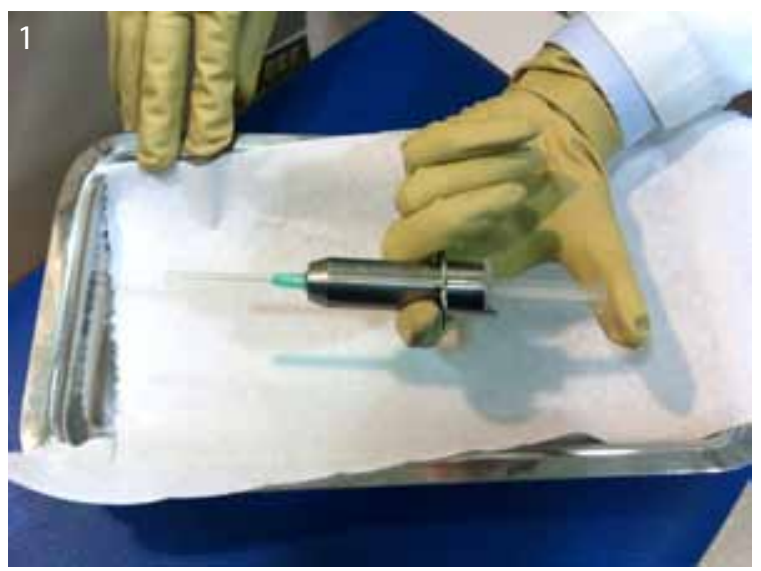

Figura 1. Parte del material utilizado durante el procedimiento, que incluye el uso de guantes plomados y escudo metálico para la jeringa.

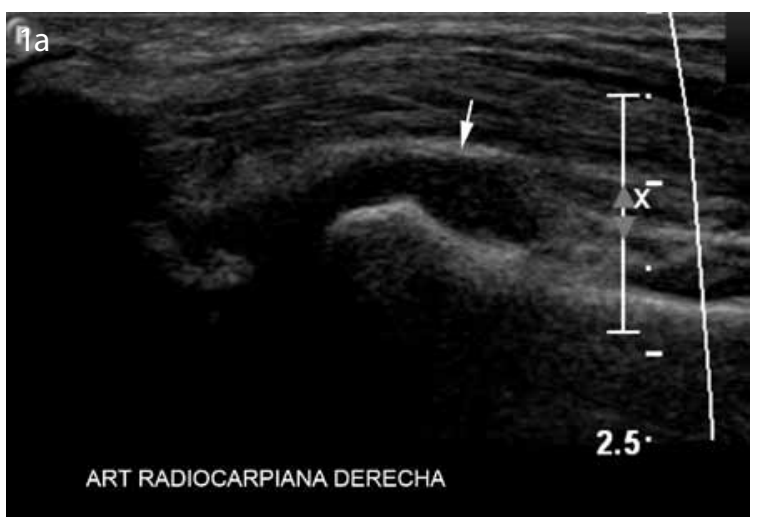

Después de la inyección del radiofármaco, es fundamental limpiar el canal de inyección para evitar la fuga del radiofármaco a los tejidos periariculares en el trayecto de la aguja, con el consiguiente riesgo de radionecrosis ${ }^{(3)}$. Esto se puede lograr tanto con la administración de corticoesteroides como de suero fisiológico.
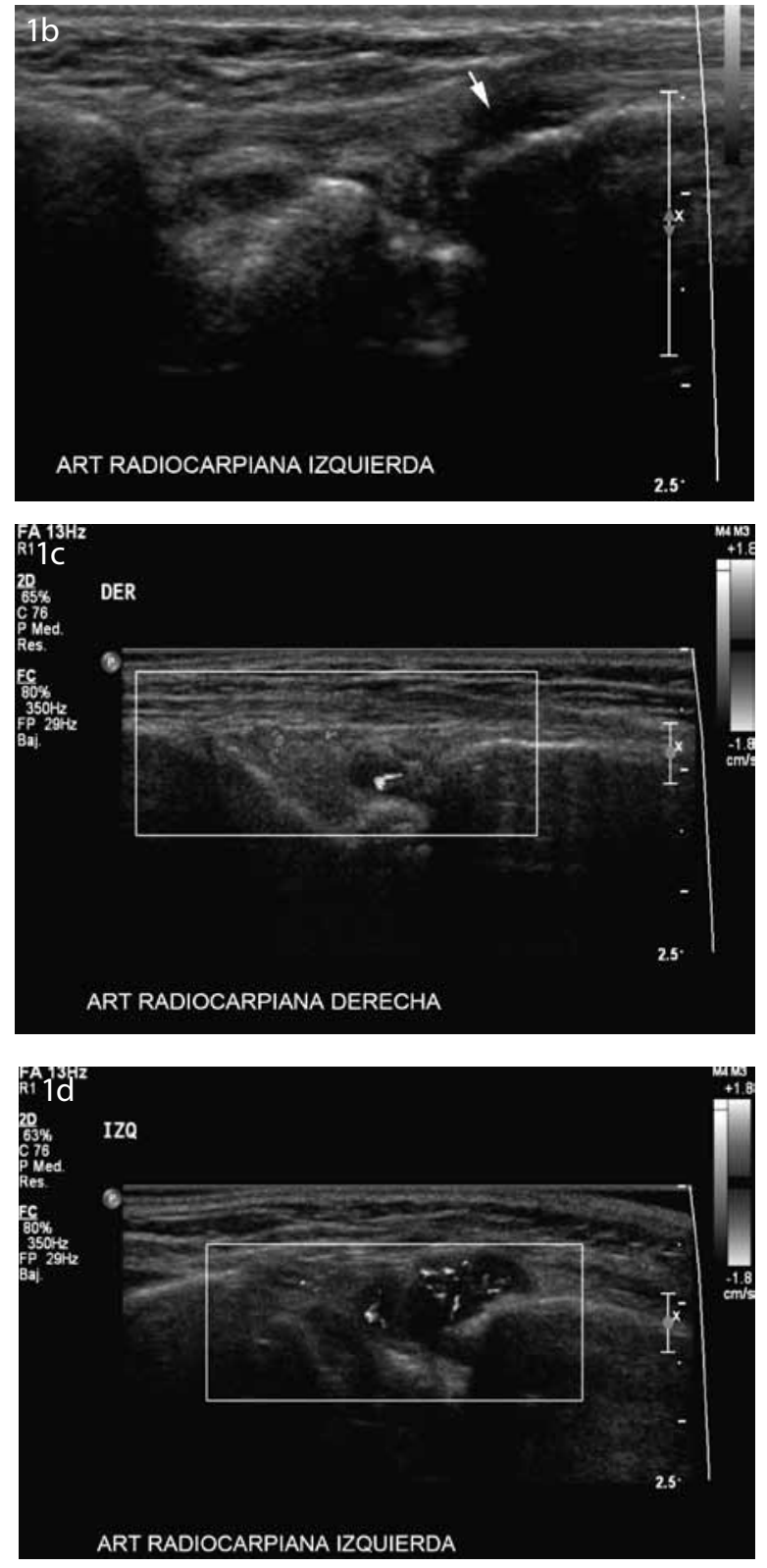

Indicaciones al paciente y evaluación post tratamiento

Se debe indicar al paciente que la mejoría en los síntomas puede demorar hasta cuatro semanas y que, incluso, puede existir aumento del dolor los primeros días secundario a sinovitis por radiación. Este efecto se puede minimizar administrando corticoesteroides junto con el radiofármaco ${ }^{(2,3)}$.

Posterior al procedimiento se debe indicar inmovilización articular por lo menos por 48 horas para minimizar el riesgo de fuga del radiofármaco (se debe considerar profilaxis de trombosis venosa profunda cuando se tratan articulaciones de la extremidad inferior).

\section{Seguridad del procedimiento}

La necrosis por radiación de los tejidos periarticulares es una complicación infrecuente secundaria 
a la fuga del radiofármaco. Suele manifestarse por aparición de úlceras cutáneas en el sitio de punción, con mala respuesta al tratamiento conservador y que requieren de debridación quirúrgica e injerto cutáneo. Se han descrito casos de radionecrosis cutánea por inyección de Re186 tratadas efectivamente con oxígeno hiperbárico ${ }^{(8)}$. Otras complicaciones descritas son osteonecrosis e infección intraarticular ${ }^{(9)}$.

\section{Reporte de caso clínico}

Paciente de sexo femenino, 21 años de edad. Artritis crónica juvenil óligoarticular diagnosticada en 2004, con compromiso predominantemente en ambas muñecas (Figura 2). En los exámenes de laboratorio destaca positividad para factor reumatoideo y anticuerpos antinucleares. Inicia tratamiento con metotrexato, con respuesta parcial. Se agregan drogas moduladoras de la enfermedad (infliximab), con ausencia de alivio sintomático, por lo que se suspende y se inicia tratamiento con etanercept el año 2007, con mejoría de la sintomatología. En octubre de 2009 presenta reactivación, con rigidez, limitación funcional y dolor diurno y nocturno en ambas muñecas que no cede a pesar del uso de analgésicos y esteroides sistémicos.
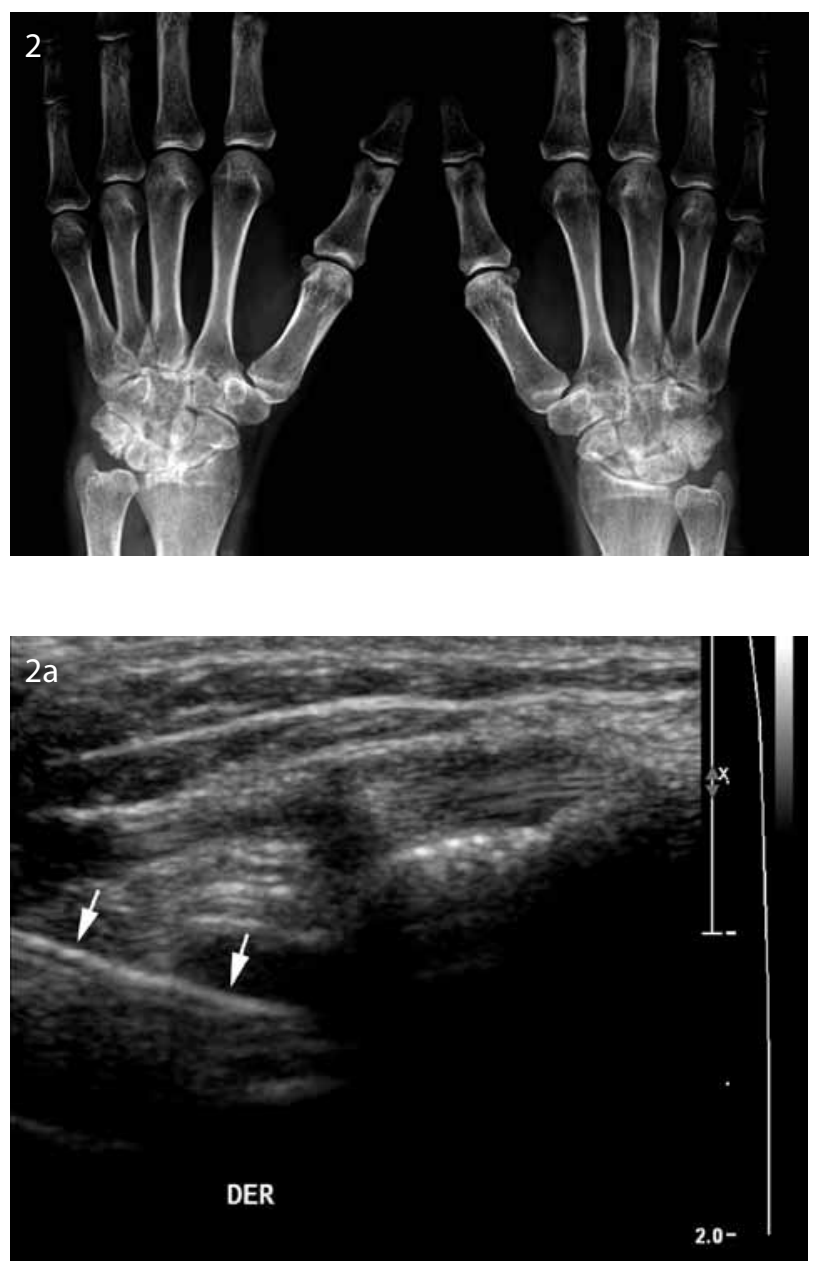

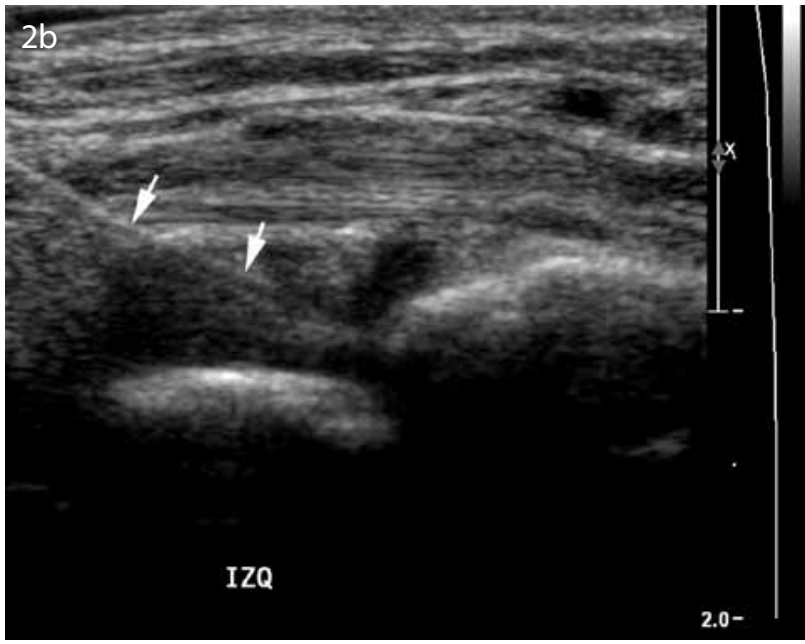

Figura 2. Radiografía de ambas muñecas, que muestra disminución de amplitud uniforme de articulaciones radio carpianas, intercarpiana y carpometacarpiana bilateral, con compromiso erosivo asociado.

En noviembre de 2009 se realizó ecotomografía de ambas muñecas, donde se evidencia moderada distensión y engrosamiento sinovial en compartimientos radiocarpianos e intercarpianos, con hiperemia al estudio Doppler color e irregularidad del contorno óseo en los huesos carpianos (Figura $3 \mathrm{a}, \mathrm{b}, \mathrm{c}, \mathrm{d}$ ). Dado el cuadro clínico y los hallazgos descritos en ecotomografía, compatibles con sinovitis erosiva con signos de actividad inflamatoria a pesar de haber optimizado la terapia sistémica, se decide realizar radiosinovectomía.

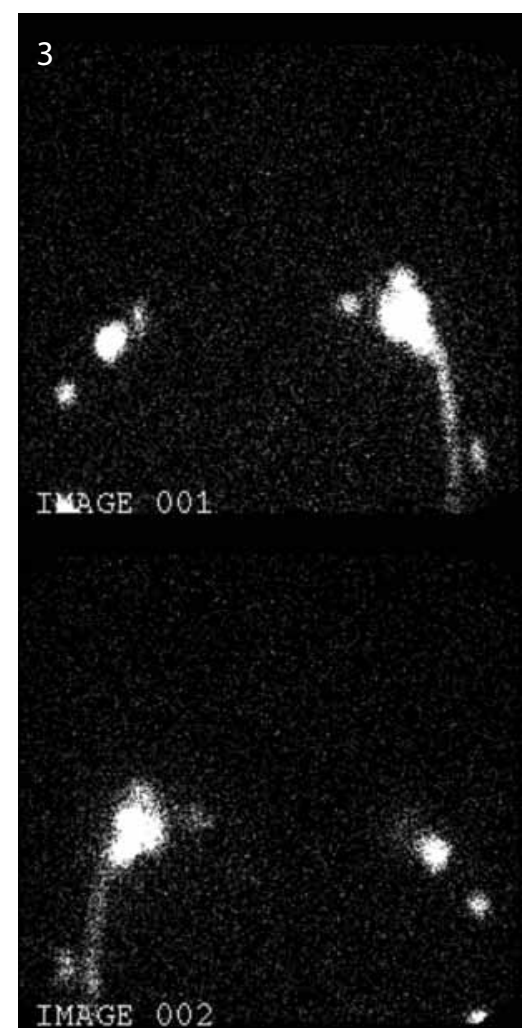



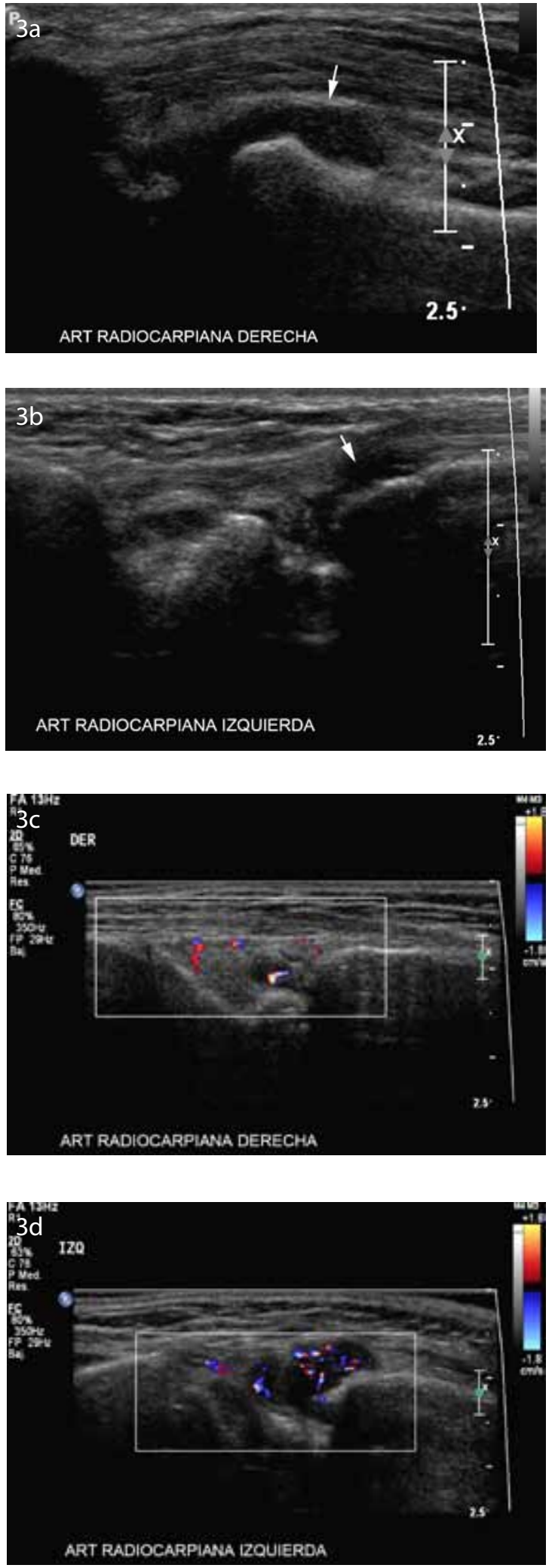

Figura 3. Imágenes de ecotomografía de muñeca derecha e izquierda ( $a$ y $b$ respectivamente), que demuestran engrosamiento sinovial y distensión con líquido de los compartimentos radiocarpiano y mediocarpiano (flechas). Al estudio Doppler (c y d), se observa aumento del flujo vascular que refleja hiperemia secundaria al compromiso inflamatorio.
En enero de 2010 se realiza inyección bajo guía ultrasonográfica (Figura 4 a, b) de 0,5 ml de solución de Re186-sulfuro coloidal (dosis de $2 \mathrm{mCi}$ en un volumen de 0,5 ml en cada muñeca) y corticoesteroides intraarticulares (solución de dipropionato y fosfato sódico de betametasona, 0,5 $\mathrm{ml}$ en muñeca derecha y $1,0 \mathrm{ml}$ en muñeca izquierda). Posteriormente se realizó estudio cintigráfico que demostró adecuada distribución del radiofármaco en los compartimentos articulares de ambas muñecas (Figura 5), sin captación significativa en ganglios axilares.
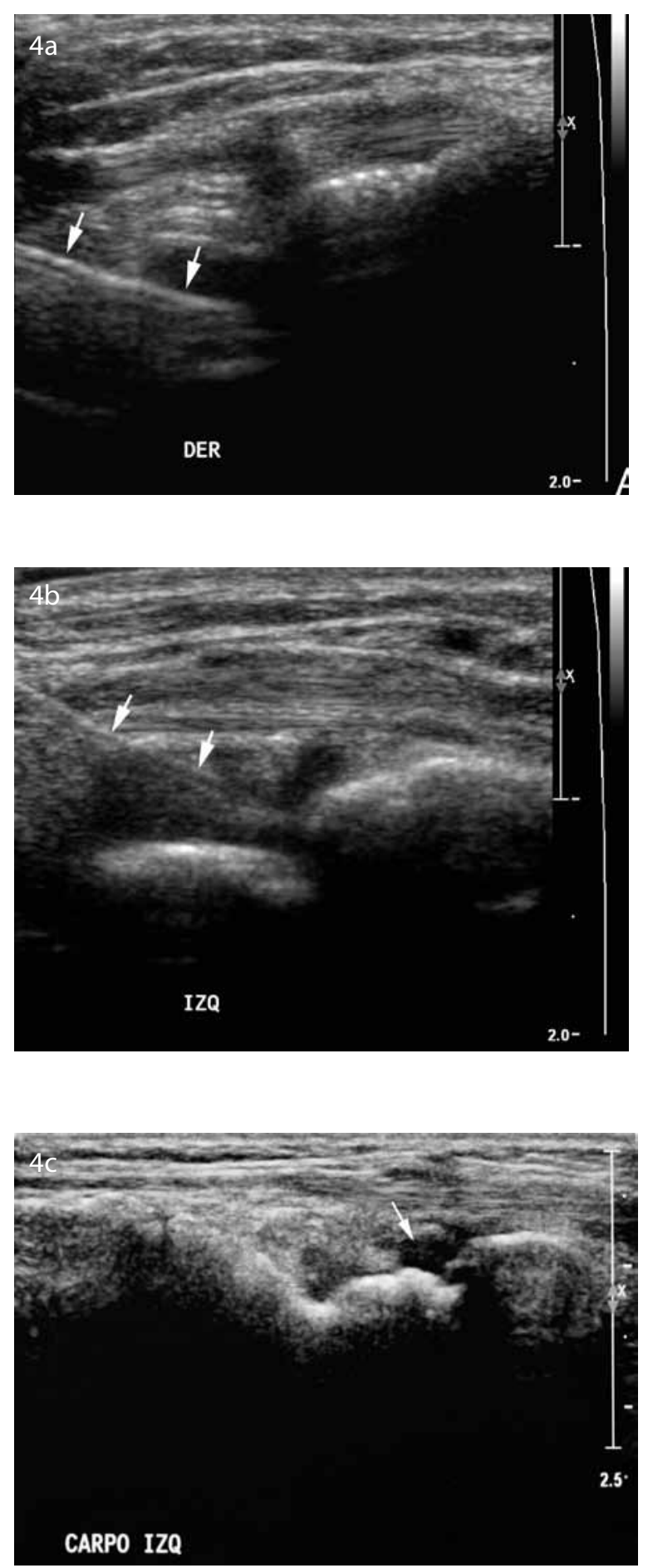


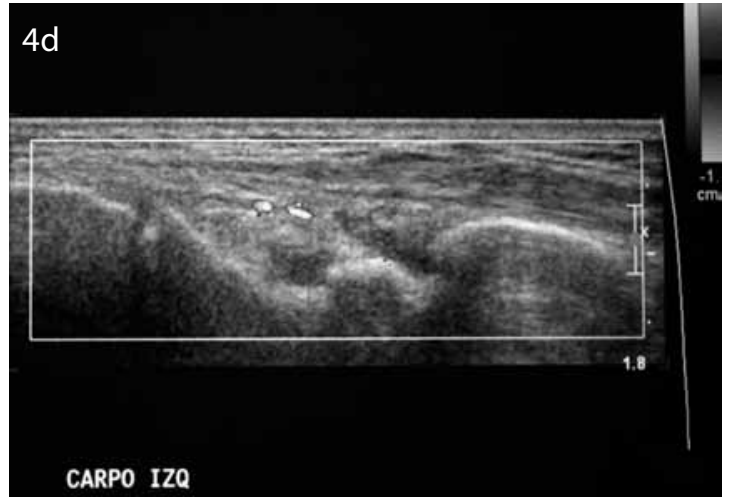

Figura 4. Imágenes de punción articular con guía ecotomográfica de muñeca derecha e izquierda ( $a$ y b respectivamente). Por vía dorsal se avanza aguja de 21 $\mathrm{G}$ (flechas) hasta el receso posterior de la articulación radiocarpiana, para posteriormente inyectar el radiofármaco y corticoesteroide. La visualización en tiempo real permite una inyección intraarticular segura.

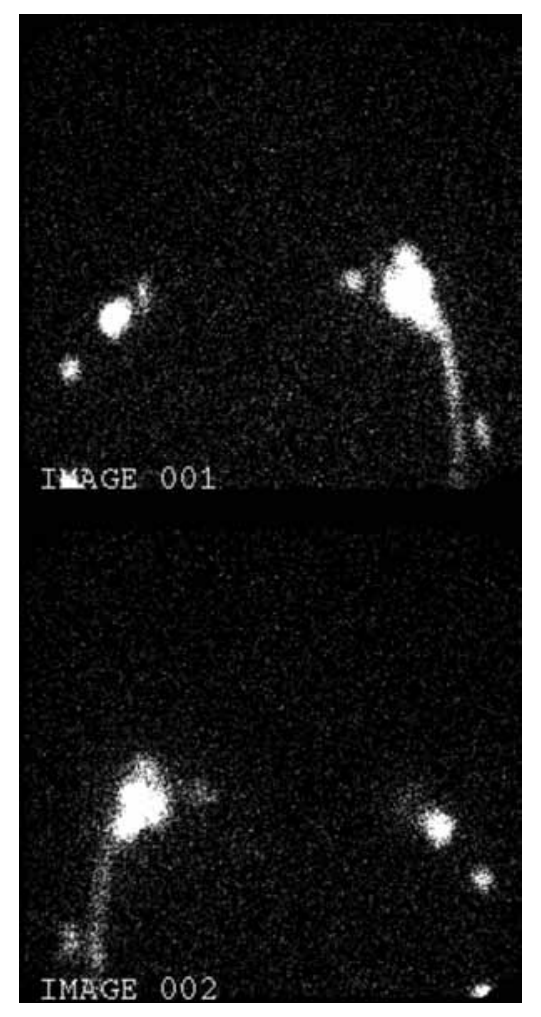

Figura 5. Imágenes de cintigrafía post-procedimiento, que demuestran intensa captación del sulfuro coloidal de renio-186 en ambas articulaciones radiocarpianas.

En control, al mes después del procedimiento, la paciente refiere significativa disminución del dolor y limitación funcional, mientras que en controles a los dos y seis meses refiere ausencia de dolor, sin signos de sinovitis al examen clínico. En septiembre de 2010 se realiza control ultrasonográfico, que demuestra ausencia de derrame articular y sinovitis a derecha y leve sinovitis residual a izquierda (Figura $6 a, b, c, d$ ).
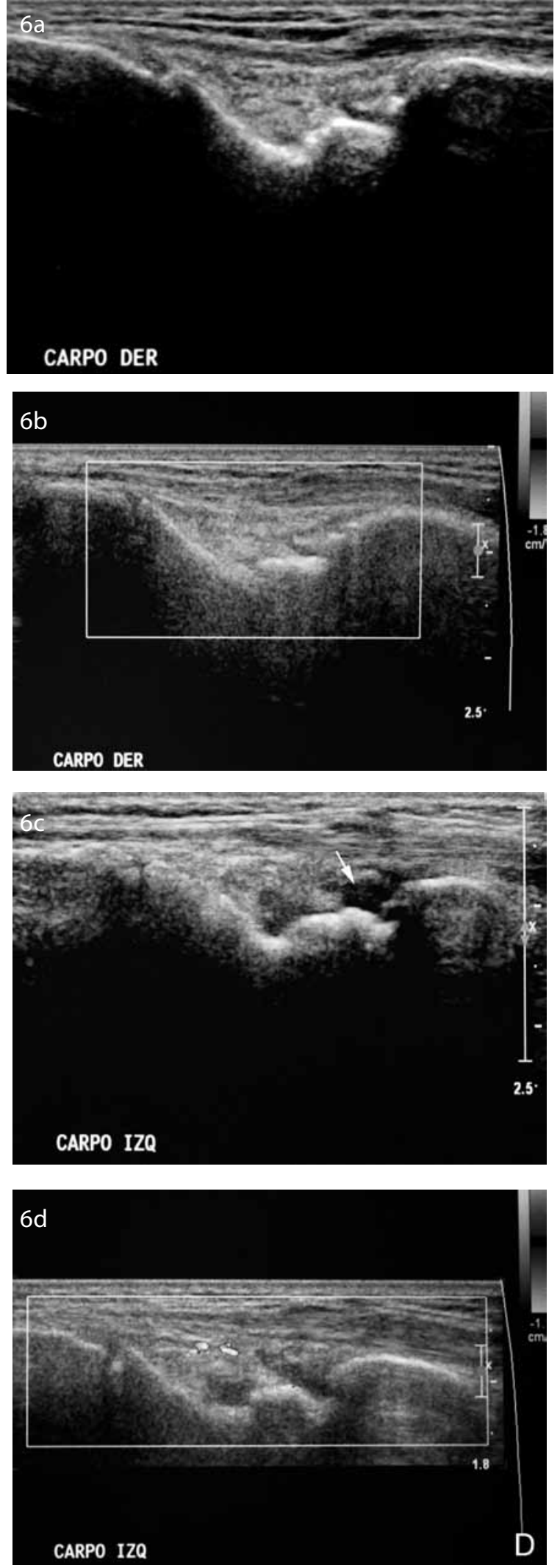

Figura 6. Imágenes de ecotomografía en escala de grises y Doppler de muñeca derecha (a y b) e izquierda (c y d); control 8 meses post tratamiento demuestra resolución del derrame articular y de la hiperemia en los compartimentos radiocarpianos e intercarpianos a derecha y mínima sinovitis residual a izquierda (flecha). 


\section{Discusión}

La radiosinovetomía con Re186 es un procedimiento seguro y eficaz cuando la indicación clínica es adecuada, se realiza por un profesional entrenado y se siguen las recomendaciones postprocedimiento. Es un tratamiento de segunda línea, que suele realizarse después del fracaso terapéutico con uso de corticoides intraarticulares, pero también es posible realizar ambos procedimientos en la misma sesión. En estudios randomizados controlados, la radiosinovectomía ha demostrado ser superior al placebo y los corticoesteroides intraarticulares en el alivio sintomático y mejoría de la limitación funcional en pacientes con artropatías inflamatorias ${ }^{(10)}$. En el estudio de Tebib y colaboradores ${ }^{(11)}$, se comparó la efectividad de la radiosinovectomía con renio versus corticoesteroides (cortivazol 31,75 mg) en 129 articulaciones (hombro, codo, muñeca, cadera y tobillo) en 81 pacientes con artritis reumatoide. Si bien no hubo diferencias estadísticamente significativas en la mejoría del dolor, edema y limitación de la movilidad en los controles a los 3, 6 y 12 meses postratamiento. A partir de los 18 meses, el grupo tratado con renio presentó significativa mayor disminución en estos tres parámetros en comparación con el grupo tratado con corticoesteroides (con disminución del dolor, edema y limitación de la movilidad a los 24 meses en el 94\%, $82 \%$ y $59 \%$ respectivamente en las articulaciones tratadas con radiosinovectomía, versus $73 \%, 53 \%$ y $42 \%$ en las que se administró corticoesteroides). El segundo grupo también presentó mayor tasa de reactivación inflamatoria a partir de los 24 meses en comparación con la radiosinovectomía. En un segundo estudio randomizado controlado por placebo se comparó la radiosinovectomía (con Re186 o Er169) más inyección de corticoesteroides versus placebo más corticoesteroides en 68 articulaciones de extremidades superiores (hombro, codo, muñeca, metacarpofalángicas e interfalángicas) en 44 pacientes con artropatías inflamatorias ${ }^{(12)}$. En el grupo de radiosinovectomía más corticoesteroides la tasa de respuesta clínica favorable fue de $67 \%$ y $71 \%$ a los 6 y 12 meses respectivamente versus $29 \%$ y $32 \%$ en el grupo de placebo más corticoesteroides. La combinación de inyección de radiofármaco junto con corticoesteroides también ha demostrado tener mayor efectividad que el uso de Re186 y corticoesteroides por separado. En el estudio de Göbel y colaboradores ${ }^{(13)}$ se comparó la efectividad del tratamiento en tres grupos de pacientes con artritis reumatoide en los que se realizó inyección de Re186, inyección de corticoesteroides (triamcinolona) o inyección combinada de Re186 y corticoesteroides. El grupo que recibió terapia combinada presentó disminución del dolor y de la sinovitis más rápidamente que el grupo en el que sólo se usó Re186 y presentó significativa disminución de los síntomas a los 3 años de segui- miento en comparación al grupo que sólo recibió corticoesteroides.

La efectividad de la radiosinovectomía parece ser mejor en extremidades superiores. En un estudio prospectivo no controlado se trataron 183 pacientes con artropatía en extremidades superiores e inferiores que presentaban sinovitis persistente a pesar de uso de corticoesteroides intraarticulares. Si bien la respuesta clínica general fue favorable en el $68 \%$ de los casos, la eficacia de la radiosinovectomía para articulaciones de extremidades superiores fue del $79 \%$, mientras que para extremidades inferiores fue de $60 \%{ }^{(14)}$.

La indicación más frecuente para la radiosinovectomía después de las artropatías inflamatorias (especialmente artritis reumatoide) es la artropatía hemofílica. El procedimiento está indicado cuando el paciente sufre de sinovitis crónica secundaria a hemartrosis recurrentes con mala respuesta a terapia hematológica. En estos pacientes es un procedimiento más seguro y económico que la sinovectomía quirúrgica, con tasas de éxito que varían entre un $64 \%$ y un $91 \%$ según los distintos estudios ${ }^{(4,10)}$.

\section{Conclusión}

La radiosinovectomía es un procedimiento seguro en manos expertas y que ofrece un significativo alivio sintomático a largo plazo especialmente en pacientes con artropatías inflamatorias con compromiso oligoarticular, con respuesta insuficiente a la terapia sistémica. La efectividad del tratamiento es mayor cuando se realiza inyección combinada de corticoesteroides y radiofármaco en articulaciones de la extremidad superior, en donde el volumen de los espacios articulares es menor en comparación con la extremidad inferior. La guía ultrasonográfica permite al profesional entrenado realizar el procedimiento sin necesidad de inyectar medio de contraste, con una administración localizada del radiofármaco y menor potencial teórico de complicaciones. El conocimiento de las indicaciones, criterios de selección de pacientes y potenciales complicaciones por parte del equipo de salud es fundamental para el éxito de esta alternativa terapéutica y la consiguiente mejoría en la calidad de vida de los pacientes.

\section{Bibliografía}

1. Fellinger K, Schmid J. Local therapy of rheumatic diseases. Wein Z Inn Med. 1952; 33: 351-363.

2. Clunie G, Fisher M. EANM procedure guidelines for radiosynovectomy. Eur J Nucl Med Mol Imaging. 2003; 30: 12-16.

3. Schneider P, Farahati J, Reiners C. Radiosynovectomy in rheumatology, orthopedics and hemophilia. J Nucl Med. 2005; 46(s): 48-54.

4. Silva M, Linás A. Chronic hemophilic synovitis: the role of radiosynovectomy. Treatment of Hemophilia, 2004; 33: 1-10. 
5. Kraft O, Kasparek R, Stepien A. Reradiosynoviorthesis of the Knee. Cancer Biother Radiopharm. 2005; 20(3): 356-362.

6. Pirich $C$, Schwameis $E$, Bernecker $P$, Radauer M, Friedl M, Lang $S$, et al. Radiation Synovectomy on Articular Cartilage, Synovial Thickness and Enhancement as Evidenced by MRI in Patients with Chronic Synovitis. J Nucl Med 1999; 40: 1277-1284.

7. Balint P, Kane D, Hunter J, Mclnnes I, Field M, Sturrock R. Ultrasound guided versus conventional joint and soft tissue fluid aspiration in rheumatology practice: a pilot study. J Rheumatol. 2002; 29(10): 2209-2213.

8. Kampen WU, Matis E, Czeck N, Soti Z, Gratz S, Henze E. Serious complications after radiosynoviorthesis. Survey on frequency and treatment modalities. Nuklearmedizin 2006; 45: 262-268.

9. Kisielinski K, Bremer D, Knutsen A, Röttger P, Fitzek J. Complications following radiosynoviorthesis in osteoarthritis and arthroplasty: Osteonecrosis and intra-articular infection. Joint Bone Spine. 2010; 77(3): 252-257.
10. Klett $R$, Lange $U$, Haas $H$, Voth M, Pinkert J. Radiosynoviorthesis of medium-sized joints with rhenium186-sulphide colloid: a review of the literature. Rheumatology. 2007; 46(10): 1531-1537.

11. Tebib JG, Manil L, Mödder G. Verrier P, et al. Better results with Rhenium-186 radiosynoviorthesis than with cortivazol in rheumatoid arthritis (RA): a two-year follow-up randomized controlled multicentric study. Clin Exp Rheum 2004; 22: 609-616.

12. Van der Zant FM, Jahangier ZN, Moolenburgh JD, Swen WA, Boer RO, Jacobs JW. Clinical effect of radiation synovectomy of the upper extremity joints: a randomized, double blind, placebo controlled study. Eur J Nucl Mol Imaging 2007; 34: 212-218.

13. Radiosynoviorthesis with rhenium-186 in rheumatoid arthritis: a prospective study of three treatment regimens. Rheumatol Int 1997; 17: 105-108.

14. Jahangier ZN, Moolenburgh JD, Jacobs JW, Serdijn $\mathrm{H}$, Bijlsma JW. The effect of radiation synovectomy in patients with persistent arthritis: a prospective study. Clin Exp Rheumatol 2001; 19: 417-424. 